\title{
INFORMED SEPARATION OF SPATIAL IMAGES OF STEREO MUSIC RECORDINGS USING SECOND-ORDER STATISTICS
}

\author{
Stanislaw Gorlow* \\ Univ. Bordeaux \\ LaBRI, UMR 5800 \\ 33400 Talence, France
}

\author{
Sylvain Marchand \\ Univ. Brest \\ Lab-STICC — CNRS, UMR 6285 \\ 29238 Brest, France
}

\begin{abstract}
In this work we address a reverse audio engineering problem, i.e. the separation of stereo tracks of professionally produced music recordings. More precisely, we apply a spatial filtering approach with a quadratic constraint using an explicit sourceimage-mixture model. The model parameters are "learned" from a given set of original stereo tracks, reduced in size and used afterwards to demix the desired tracks in best possible quality from a preexisting mixture. Our approach implicates a side-information rate of $10 \mathrm{kbps}$ per source or channel and has a low computational complexity. The results obtained for the SiSEC 2013 dataset are intended to be used as reference for comparison with unpublished approaches.
\end{abstract}

Index Terms - Informed source separation, low-order statistics, professionally produced music recordings, spatial filtering, stereo images

\section{INTRODUCTION}

Most if not all of today's professionally produced music has undergone two basic processes: mixing and mastering. Many established music distribution formats, moreover, are strictly stereo. While in the mastering stage the final mix is prepared and transfered to a data storage device, mixing represents the process that ends up in a summation of individually recorded and edited audio from distinct mono or stereo sources into a composite stereo mixture. The apparent placement of sources between the speakers in a stereo sound field is also known as "imaging" [1] in professional audio engineering. The notion of spatial "images" in a source separation context can e.g. be found in [2]. The separation of stereo images of individual or grouped sources is the central point of the present paper.

For the reason that the total number of source channels is usually greater than the number of mixture channels, mixing is mathematically underdetermined. So, demixing constitutes an ill-posed source separation problem that cannot be solved without additional assumptions or prior information. We use

\footnotetext{
* This research was partially funded by the "Agence Nationale de la Recherche" within the scope of the DReaM project (ANR-09-CORD-006).
}

the knowledge of the mixing process and low-order statistics of the sources as additional information for our algorithm in order to find the optimal solution. The content of the paper is therefore an extension to our previous work on the informed separation of mono sources [3]. We introduce a more general source-image signal model based on common studio practice and also generalize the mixture model to a sum of images of mono and stereo sources. The demixing problem is likewise addressed in an informed source separation context [4]. With the proposed approach one can decompose the final mix into distinct tracks or into the background and foreground objects and in the same manner one can separate the vocal from the instrumental track for karaoke.

The organization of the paper is as follows. The problem at hand is given in Section 2. Section 3 illustrates the sourceimage signal model, the estimation of model parameters, and how the latter can be reduced in size. The extended mixture model is discussed in Section 4. There it is also shown how a source of interest and its image are separated using a linearly constrained spatial filter. The proposed approach is evaluated on five multitracks of changing sound complexity in Section 5. Section 6 concludes the paper with an outlook.

\section{PROBLEM STATEMENT}

The problem at hand is stated as follows. Given access to the original stereo images of distinct sources, recover a subset of the images in best possible quality from a mixture composed of the original images using a source-image-mixture model. The model parameters shall be estimated from the accessible image signals and used during recovery. The amount of data associated with the model parameters should furthermore be kept to a minimum.

\section{PARAMETRIC ANALYSIS}

\subsection{Source-image model}

We model the signals in the complex subband domain. Each subband signal is said to be a zero-mean circular symmetric 
complex Gaussian stochastic process that evolves over time $n$. The set of subband signal components at a given instant $n$ of a single source is deemed to be mutually independent, and so is the set of sources. The sources are thus uncorrelated. A source may be mono or stereo. The two channels of a stereo source are treated as two separate mono sources. So, a stereo source can be thought of in terms of a centered spatial image of an acoustic source that was recorded with two independent microphones.

A mono source is assigned a location in the stereo sound field via pan control, whereas a stereo source or its centered image is positioned via balance control:

$$
\begin{aligned}
\mathbf{u}_{i}(n) & =a_{i l} \mathbf{e}_{l} s_{i l}(n)+a_{i r} \mathbf{e}_{r} s_{i r}(n) \\
& =\mathbf{a}_{i} \circ \mathbf{s}_{i}(n),
\end{aligned}
$$

where $\circ$ denotes the Hadamard or entrywise product between the time-invariant steering vector $\mathbf{a}_{i}=\left[\begin{array}{ll}a_{i l} & a_{i r}\end{array}\right]^{\top}$ and the $i$ th stereo source $\mathbf{s}_{i}=\left[\begin{array}{ll}s_{i l} & s_{i r}\end{array}\right]^{\top}$. In the case of a mono source, $s_{i l}=s_{i r}=s_{i}$. Accordingly, $\mathbf{u}_{i}$ represents the stereo image of the $i$ th source. In (1), $\left\{\mathbf{e}_{l}, \mathbf{e}_{r}\right\}$ is the standard basis of $\mathbb{R}^{2}$, $\mathbf{a}_{i} \in \mathbb{R}^{2}$ and $\mathbf{s}_{i}(n) \in \mathbb{C}^{2}$. The subband index $k$ is omitted for simplicity. The $i$ th steering vector $\mathbf{a}_{i}$ is defined as

$$
\mathbf{a}_{i} \triangleq \frac{\mathbf{a}_{i}}{\left\|\mathbf{a}_{i}\right\|}=\left[\begin{array}{c}
\sin \theta_{i} \\
\cos \theta_{i}
\end{array}\right],
$$

in the case of a mono source, or else as

$$
\mathbf{a}_{i} \triangleq \frac{\mathbf{a}_{i}}{a_{i, \mathrm{ref}}}
$$

where

$$
a_{i, \mathrm{ref}}= \begin{cases}a_{i l} & \text { if } a_{i l} \geqslant a_{i r}, \\ a_{i r} & \text { otherwise }\end{cases}
$$

in the case of a stereo source.

\subsection{Model parameter estimation}

Consider the stereo image of a distinct source as given. From the stereo signal, we can estimate the model parameters that are used as prior information for source separation, which is detailed in Section 4. These parameters describe the source's location and how the signal power or variance is distributed over time and frequency. We apply the following protocol.

First, we compute the zero-lag cross-covariance between the left and the right channel and normalize it by the product of average power in each channel using the root mean square (RMS) as measure:

$$
\operatorname{corr}\left(u_{i l}, u_{i r}\right)=\frac{\operatorname{cov}\left(u_{i l}, u_{i r}\right)}{\operatorname{RMS}_{i l} \mathrm{RMS}_{i r}},
$$

where the sample covariance is defined as

$$
\operatorname{cov}\left(u_{i l}, u_{i r}\right)=\frac{1}{N} \sum_{n=1}^{N} u_{i l}(n) u_{i r}^{*}(n)
$$

with $*$ denoting complex conjugation. The RMS is given by

$$
\operatorname{RMS}_{i j}=\sqrt{\frac{1}{N} \sum_{n=1}^{N} u_{i j}^{2}(n) .}
$$

In our case, corr is identical with Pearson's correlation. The computation of (5) may be carried out in either the subband domain or the time domain. In the latter case, $u_{i j}(n)$ is real. The sample size $N$ corresponds to the duration of the signal over which it can be considered (wide-sense) stationary and ergodic. The correlation coefficient may also be computed on a sample basis assuming non-stationarity. Then, if the sample variance

$$
\begin{aligned}
& \operatorname{var}\left\{\operatorname{corr}_{n}\left(u_{i l}, u_{i r}\right)\right\} \\
& \quad=\frac{1}{N} \sum_{n=1}^{N}\left[\operatorname{corr}_{n}\left(u_{i l}, u_{i r}\right)-\overline{\operatorname{corr}}_{n}\left(u_{i l}, u_{i r}\right)\right]^{2} \\
& \quad \rightarrow 0
\end{aligned}
$$

where

$$
\overline{\operatorname{corr}}_{n}\left(u_{i l}, u_{i r}\right)=\frac{1}{N} \sum_{n=1}^{N} \operatorname{corr}_{n}\left(u_{i l}, u_{i r}\right),
$$

the source is considered as mono and its respective pan angle is estimated according to

$$
\hat{\theta}_{i}=\operatorname{arccot} \frac{\mathrm{RMS}_{i r}}{\mathrm{RMS}_{i l}}
$$

where arccot is the arccotangent. In the reverse case, that is if the source is stereo, its power balance is estimated as

$$
\hat{a}_{i, \neg \mathrm{ref}}=\frac{\mathrm{RMS}_{i, \neg \mathrm{ref}}}{\operatorname{RMS}_{i, \mathrm{ref}}} \quad \text { with } a_{i, \mathrm{ref}}=1,
$$

where ref $\in\{l, r\}$ is the channel with the greater RMS value and $\neg$ ref is the complementary channel.

The mean signal power or variance of a subband signal is formally given by ( $k$ is the subband index)

$$
\phi_{i j k}=\mathrm{E}\left[\left|s_{i j k}\right|^{2}\right],
$$

where $\mathrm{E}$ is the statistical expectation. When using the shorttime Fourier transform (STFT) for the analysis of speech and audio, stationarity and ergodicity are usually assumed for the time-domain segment that is time-frequency transformed [3]. The resulting subband signals are non-stationary, and so $\phi_{i j k}$ $\rightarrow \phi_{i j k}(n)$, i.e. the subband variance is instantaneous. Thus, $\left\{\phi_{i j k}(n)\right\}_{k}$ is likewise the short-time power spectral density (STPSD) of the $j$ th channel at instant $n$. For a mono source, $\phi_{i j k}=\phi_{i k}$. The STPSD describes the variance distribution of the corresponding time-domain segment over frequency. It is estimated in the STFT domain according to

$$
\hat{\phi}_{i j k}(n)=\left|S_{i j}(k, n)\right|^{2} \quad \text { with } j \in\{l, r\}
$$


or $\hat{\phi}_{i k}(n)=\left|S_{i}(k, n)\right|^{2}$, where $\left\{S_{i l}(k, n), S_{i r}(k, n)\right\}_{k}$ is the spectrum of a stereo source and $\left\{S_{i}(k, n)\right\}_{k}$ the spectrum of a mono source, respectively. Individual spectra are obtained from the corresponding images by

$$
\hat{S}_{i}(k, n)=\left[\sin \hat{\theta}_{i} \quad \cos \hat{\theta}_{i}\right] \mathbf{u}_{i k}(n),
$$

in the case of a mono source, or else by

$$
\hat{S}_{i, \mathrm{ref}}(k, n)=u_{i, \mathrm{ref}, k}(n)
$$

and

$$
\hat{S}_{i, \neg \mathrm{ref}}(k, n)= \begin{cases}\frac{u_{i, \neg \mathrm{ref}, k}(n)}{\hat{a}_{i, \neg \mathrm{ref}}} & \text { if } \hat{a}_{i, \neg \mathrm{ref}} \neq 0, \\ 0 & \text { otherwise, }\end{cases}
$$

in the case of a stereo source.

\subsection{Parameter quantization and coding}

Here, we present one possible coding strategy that serves the reduction of side information. The discussion of the strategy is outside the scope of the paper. Our objective is to propose a scalable coding scheme with a compact representation of the metadata. Other methods, especially for spectrogram coding, can be found in [5], e.g.

The pan angle $\theta$ of a mono source is rounded to the nearest integer value using a mid-tread uniform quantizer defined as

$$
Q(x)=\Delta \cdot\left\lfloor\frac{x}{\Delta}+\frac{1}{2}\right\rfloor,
$$

where $\Delta$ is the step size and $\lfloor\cdot\rfloor$ represents the floor function. The balance parameter $a_{i, \neg \text { ref }}$ for a stereo source is encoded using an $A$-law or $\mu$-law compressor together with a uniform quantizer as in (16). For a given input $x \in[0,1]$, the $A$-law compressor output is

$$
C_{A}(x)= \begin{cases}\frac{A \cdot x}{1+\log A} & \text { if } x<\frac{1}{A} \\ \frac{1+\log (A \cdot x)}{1+\log A} & \text { otherwise }\end{cases}
$$

where $A$ is the compression parameter and $\log$ is the natural logarithm. The output of the $\mu$-law compressor is

$$
C_{\mu}(x)=\frac{\log (1+\mu \cdot x)}{\log (1+\mu)}
$$

where $\mu$ is the associated compression parameter. Using $A$ law or $\mu$-law compression, the signal-to-noise ratio (SNR) is kept constant over almost the entire range of $a_{i, \neg \text { ref }}$ [6]. The STPSD of a mono source (or a channel of a stereo source) is quantized on an ERB-like frequency scale according to

$$
\bar{\phi}_{i z}(n)=\frac{1}{\mathrm{ub}(z)-\mathrm{lb}(z)+1} \sum_{k=\mathrm{lb}(z)}^{\mathrm{ub}(z)} \hat{\phi}_{i k}(n),
$$

where $\operatorname{lb}(z)=\inf \left\{k \mid z_{k}=z\right\}, \operatorname{ub}(z)=\sup \left\{k \mid z_{k}=z\right\}$, $z$ is the quantization index and

$$
z_{k}=\left\lfloor 21.4 \log _{10}\left(4.37 f_{s} / N k+1\right)\right\rfloor .
$$

In (20), $f_{s}$ is the sampling rate and $N$ is the STFT size. The average power values are then converted from linear scale to logarithmic scale and quantized using (16). These values are encoded using differential pulse-code modulation (DPCM) in combination with Huffman coding. The difference between adjacent power values is calculated in the direction of time or frequency or between channel pairs depending on what gives the lowest entropy.

\section{SEPARATION OF STEREO IMAGES}

\subsection{Mixture model and spatial covariance matrix}

The mixture is considered to be obtained by superposition of distinct stereo images that were created according to (1). To account for professionally produced music recordings, $\mathbf{s}_{i}(n)$ is regarded as having undergone prior processing in the form of linear and nonlinear audio effects [7]. The mixture signal is thus formulated as

$$
\begin{aligned}
\mathbf{x}_{k}(n) & =\sum_{i \in \mathrm{I}} \overbrace{\mathbf{a}_{i} \circ \mathbf{s}_{i k}(n)}^{\mathbf{u}_{i k}(n)} \\
& =\sum_{p \in \mathrm{P}} \mathbf{a}_{p} s_{p k}(n)+\sum_{q \in \mathrm{Q}} \mathbf{a}_{q} \circ \mathbf{s}_{q k}(n),
\end{aligned}
$$

where set $\mathrm{P}=\left\{i \in \mathrm{I} \mid \forall n\left[s_{i l k}(n)=s_{\text {irk }}(n)\right]\right\}$ represents the mono sources, while $\mathrm{Q}=\left\{i \in \mathrm{I} \mid \exists n\left[s_{i l k}(n) \neq s_{\text {irk }}(n)\right]\right\}=$ I $\backslash P$ represents the stereo sources, respectively.

The local mixture spatial covariance matrix is given by

$$
\begin{aligned}
\mathbf{R}_{\mathbf{x} \mathbf{x}, k}(n)= & \mathrm{E}\left[\mathbf{x}_{k}(n) \mathbf{x}_{k}^{\mathrm{H}}(n)\right] \\
= & \sum_{p \in \mathrm{P}} \mathbf{a}_{p} \mathbf{a}_{p}^{\top} \phi_{p k}(n) \\
& \quad+\sum_{q \in \mathbf{Q}} \mathbf{a}_{q} \mathbf{a}_{q}^{\top} \circ \mathbf{\Phi}_{q k}(n),
\end{aligned}
$$

where $\left\{\phi_{p k}(n)\right\}_{k}$ is the $p$ th mono source's STPSD and

$$
\boldsymbol{\Phi}_{q k}(n)=\left[\begin{array}{ll}
\phi_{q, l l, k}(n) & \phi_{q, l r, k}(n) \\
\phi_{q, l r, k}^{*}(n) & \phi_{q, r r, k}(n)
\end{array}\right],
$$

where $*$ denotes complex conjugation. In (23), $\left\{\phi_{q, l l, k}(n)\right\}_{k}$ and $\left\{\phi_{q, r r, k}(n)\right\}_{k}$ are the $q$ th stereo source's left- and rightchannel STPSDs, while $\left\{\phi_{q, l r, k}(n)\right\}_{k}$ is the short-time cross spectral density (STCSD).

In the next section it is shown how the summation of the stereo images can be "undone" by means of spatial filtering. There, in (26) and (30), the inverse of $\mathbf{R}_{\mathbf{x x}, k}(n)$ needs to be computed. The condition number of a matrix $\kappa$ is often used 
to estimate how accurate the solution will be in the presence of observation errors in $\mathbf{x}_{k}(n)$. When $\mathbf{x}_{k}(n)$ is noise-free, as in our case, one may also use the condition number to argue that the accuracy of the separation of sources, which is based on spatial decorrelation, improves when the left and the right channels of a stereo source are uncorrelated. In that case, the condition number

$$
\kappa\left[\mathbf{R}_{\mathbf{x x}, k}(n)\right]=\left|\frac{\lambda_{\max }\left[\mathbf{R}_{\mathbf{x x}, k}(n)\right]}{\lambda_{\min }\left[\mathbf{R}_{\mathbf{x x}, k}(n)\right]}\right|,
$$

where $\lambda_{\max }$ and $\lambda_{\min }$ are the two eigenvalues of $\mathbf{R}_{\mathbf{x x}, k}(n)$, has the lowest value. Also in our experience, nonzero crosscovariance terms lead to a degradation of sound quality with regard to underdetermined mixtures. As a result, (23) can be simplified as

$$
\boldsymbol{\Phi}_{q k}(n)=\operatorname{diag}\left[\phi_{q l k}(n), \phi_{q r k}(n)\right]
$$

Using (22) and (25), $\mathbf{R}_{\mathbf{x x}, k}(n)$ can be reconstructed from the mixing coefficients and the STPSDs, $\hat{a}_{i}$ and $\left\{\hat{\phi}_{i j k}(n)\right\}_{k}, i=$ $1,2, \ldots, I$.

\subsection{Image separation of a mono source}

Let us assume that there are more than two active sources in a time-frequency (TF) point $(k, n)$. In this case, a mono source component is separated from the mixture signal with the aid of the power-conserving minimum-variance (PCMV) spatial filter [3]

$$
\hat{\mathbf{w}}_{p k}(n)=\mathbf{R}_{\mathbf{x x}, k}^{-1}(n) \mathbf{a}_{p} \sqrt{\frac{\phi_{p k}(n)}{\mathbf{a}_{p}^{\top} \mathbf{R}_{\mathbf{x x}, k}^{-1}(n) \mathbf{a}_{p}}}
$$

according to

$$
\hat{s}_{p k}(n)=\hat{\mathbf{w}}_{p k}^{\top}(n) \mathbf{x}_{k}(n) .
$$

The corresponding image component estimate is

$$
\hat{\mathbf{u}}_{p k}(n)=\mathbf{a}_{p} \hat{s}_{p k}(n) .
$$

If the number of active sources is at most two, the demixing becomes trivial given that the mixing system is known.

\subsection{Image separation of a stereo source}

A stereo source component is separated from the mixture in a similar manner, where the left-channel and the right-channel components are estimated simultaneously according to

$$
\hat{\mathbf{s}}_{q k}(n)=\hat{\mathbf{W}}_{q k}^{\top}(n) \mathbf{x}_{k}(n)
$$

with the PCMV spatial filter matrix being

$$
\begin{aligned}
& \hat{\mathbf{W}}_{q k}(n)=\mathbf{R}_{\mathbf{x x}, k}^{-1}(n) \boldsymbol{\Phi}_{q k}^{1 / 2}(n) \\
& \cdot \operatorname{diag}\left\{\left[\mathbf{R}_{\mathbf{x x}, k}^{-1}(n)\right]_{l l},\left[\mathbf{R}_{\mathbf{x} \mathbf{x}, k}^{-1}(n)\right]_{r r}\right\}^{-1 / 2}
\end{aligned}
$$

On the analogy of (28), the corresponding image component estimate is given by

$$
\hat{\mathbf{u}}_{q k}(n)=\mathbf{a}_{q} \circ \hat{\mathbf{s}}_{q k}(n) .
$$

From (25) and (30), it can be seen that when multiple stereo sources are present in the mixture, their component estimates exhibit the same phase between different sources. Only their spectral envelopes are shaped differently. Furthermore, when the mixture is a combination of stereo sources only, $\hat{\mathbf{W}}_{q k}(n)$ in (30) is diagonal. As a result, $s_{q j k}(n)$ is separated from the respective mixture channel using the mono PCMV filter:

$$
\hat{s}_{q j k}(n)=\sqrt{\frac{\phi_{q j k}(n)}{\sum_{i \in \mathrm{I}} a_{i j}^{2} \phi_{i j k}(n)}} x_{j k}(n) .
$$

\section{PERFORMANCE EVALUATION}

In this section, we evaluate our approach by applying it to a subset of professionally produced music recordings from the SiSEC 2013 [8] dataset. The task is to decompose an artistic mixture into a subset of constituent images that represent the sources of interest alias foreground objects and the image of the background-where applicable. The term "background" refers to the sum of background objects. The original images are given as a reference.

\subsection{Performance metrics}

We use the evaluation criteria suggested by the SiSEC 2013 committee. These include the performance metrics from the PEASS toolkit $[9,10]$ and the decoder runtime in seconds per CPU clock rate in GHz. For every mixture, we also give the side-information rate. Furthermore, we include PEMO-Q [11, 12] in our evaluation.

\subsection{Experimental design}

We use the following testing framework. With respect to the STFT, we employ a 2048-point fast Fourier transform (FFT) with a Kaiser-Bessel derived window of the same length and a 50-\% overlap between succeeding frames. The pan angle $\hat{\theta}$ is quantized and coded with 7 bits, while the balance $\hat{a}_{\neg \text { ref }}$ is quantized with 16 bits using the $A$-law compander with an $A$ of 87.6. The STPSD is quantized with 6 bits per power value using a 76-band nonuniform frequency scale. The probability mass function of the difference between contiguous STPSD values is modeled with a Laplace $(\mu, b)$ distribution with $\hat{\mu}=$ -0.2 and $\hat{b}=2$. The simulations are run in MATLAB on an Intel Core i5-520M 2.4-GHz CPU.

\subsection{Experimental results}

The results of the experiment are summarized in Table 1. As can be observed, the image-to-spatial distortion ratio (ISR) is 


\begin{tabular}{|c|c|c|c|c|c|c|c|c|c|c|c|c|}
\hline Track & Type & $\hat{\theta}$ or $\hat{a}_{\neg \mathrm{ref}}$ & SDR & ISR & SIR & SAR & OPS & TPS & IPS & APS & $\mathrm{PSM}_{t}$ & ODG \\
\hline Vocal & stereo & 0.89 & 9.76 & 16.8 & 11.5 & 21.7 & 0.38 & 0.61 & 0.68 & 0.79 & 0.76 & -2.96 \\
\hline Drums & stereo & 1.00 & 8.72 & 12.4 & 13.3 & 19.5 & 0.25 & 0.86 & 0.66 & 0.05 & 0.34 & -3.30 \\
\hline Guitar & stereo & 0.96 & 9.26 & 16.3 & 10.1 & 23.4 & 0.34 & 0.52 & 0.47 & 0.67 & 0.76 & -2.97 \\
\hline \multicolumn{13}{|c|}{ "The Ones We Love" by Another Dreamer - 59.6 kbps - 10.6 s GHz } \\
\hline Vocal & stereo & 0.99 & 8.35 & 17.1 & 9.31 & 20.9 & 0.19 & 0.54 & 0.62 & 0.86 & 0.74 & -3.00 \\
\hline Bass & mono & 45.0 & 8.60 & \begin{tabular}{|l|}
24.2 \\
\end{tabular} & 8.82 & \begin{tabular}{|l|}
27.7 \\
\end{tabular} & 0.38 & 0.62 & 0.52 & 0.34 & 0.54 & -3.21 \\
\hline Piano & stereo & 0.83 & 3.11 & 6.92 & 4.14 & 17.4 & 0.44 & 0.63 & 0.51 & 0.60 & 0.80 & -2.88 \\
\hline Background & stereo & 0.94 & 4.74 & 8.33 & 8.17 & 18.1 & 0.47 & 0.60 & 0.58 & 0.59 & 0.69 & -3.07 \\
\hline \multicolumn{13}{|c|}{ "Roads" by Bearlin $-69.8 \mathrm{kbps}-7.4 \mathrm{~s} \mathrm{GHz}$} \\
\hline Vocal & stereo & 0.90 & 9.15 & 15.5 & 10.8 & 19.5 & 0.76 & 0.62 & 0.86 & 0.68 & 0.81 & -2.82 \\
\hline Drums & stereo & 0.99 & 5.15 & 6.66 & 7.07 & 15.2 & 0.27 & 0.79 & 0.64 & 0.10 & 0.40 & -3.28 \\
\hline Bass & mono & 45.0 & 5.59 & \begin{tabular}{|l|}
18.5 \\
\end{tabular} & 5.24 & \begin{tabular}{|l|}
21.6 \\
\end{tabular} & 0.30 & 0.80 & 0.47 & 0.07 & -0.10 & -3.38 \\
\hline Claps & stereo & 0.99 & 8.92 & 13.8 & 11.9 & 20.6 & 0.05 & 0.96 & 0.67 & 0.00 & -0.03 & -3.37 \\
\hline Background & stereo & 0.97 & 4.76 & 10.6 & 5.80 & 14.9 & 0.46 & 0.62 & 0.51 & 0.60 & 0.72 & -3.03 \\
\hline \multicolumn{13}{|c|}{ "Remember the Name" by Fort Minor $-82.2 \mathrm{kbps}-13.0 \mathrm{~s} \mathrm{GHz}$} \\
\hline Vocal & mono & 47.9 & 14.5 & \begin{tabular}{|l|}
23.0 \\
\end{tabular} & 15.9 & 27.6 & 0.53 & 0.56 & 0.88 & 0.87 & 0.85 & -2.66 \\
\hline Guitar & stereo & 0.97 & 14.8 & 17.4 & 20.5 & 27.1 & 0.56 & 0.98 & 0.77 & 0.81 & 0.88 & -2.53 \\
\hline \multicolumn{13}{|c|}{ "Que Pena/Tanto Faz" by Tamy $-31.8 \mathrm{kbps}-5.8 \mathrm{~s} \mathrm{GHz}$} \\
\hline Vocal & stereo & 1.00 & 6.77 & 14.5 & 7.48 & 20.2 & 0.63 & 0.72 & 0.77 & 0.56 & 0.76 & -2.96 \\
\hline Drums & stereo & 0.97 & 8.39 & 14.6 & 10.2 & 19.9 & 0.49 & 0.82 & 0.66 & 0.34 & 0.53 & -3.22 \\
\hline Bass & stereo & 0.93 & 5.22 & 11.9 & 5.87 & 16.3 & 0.32 & 0.53 & 0.52 & 0.30 & 0.24 & -3.32 \\
\hline Background & stereo & 0.93 & 4.61 & 11.8 & 4.79 & 17.7 & 0.40 & 0.61 & 0.59 & 0.70 & 0.77 & -2.94 \\
\hline
\end{tabular}

Table 1. The SiSEC 2013 development set used in the experiment and the obtained results. The ISR, SAR and TPS values for mono sources are framed.

between 6.66 and $17.4 \mathrm{~dB}$ for a stereo source, and is greater or equal to $18.5 \mathrm{~dB}$ for a mono source. Similarly, the highest source-to-artifacts ratio (SAR) is obtained for a mono source, which is $27.7 \mathrm{~dB}$. The target-related perceptual score (TPS) shows a weak correlation not only with both the ISR and the SAR, but also with PEMO-Q's perceptual similarity measure $\mathrm{PSM}_{t}$, which then again does not take spatial hearing effects into account. The lowest TPS is at $52 \%$. The measured sideinformation rate is around $10 \mathrm{kbps}$ per mono source or stereo channel. The execution time of the algorithm is low and also faster than real time.

Table 2 compares the performance of our system with the figures reported in SiSEC 2011 for two oracle systems [13]. Their performance figures provide an upper bound for binary masking-based systems. In Table 2, positive delta values are in boldface. A significant improvement can be noticed for all items with regard to the SAR, going up to $22.4 \mathrm{~dB}$. The TPS is also higher in most cases, and so is the signal-to-distortion ratio (SDR).

\section{CONCLUSION}

In this paper we presented an extension to our previous work on the informed separation of audio sources. By generalizing the mixture model to a sum of stereo images, we have shown how a particular source of interest or its image can be filtered out from a stereo mixture using prior information. From our source-image model we inferred that the pursued approach is most effective when the foreground objects are in mono and only the background object is in stereo, which was validated in our experiment.

\section{REFERENCES}

[1] D. Gibson, The Art of Mixing: A Visual Guide to Recording, Engineering, and Production. MixBooks, LLC, 1997, ch. 2.

[2] N. Q. K. Duong, E. Vincent, and R. Gribonval, "Under- 


\begin{tabular}{lrrrrrrrr}
\hline Track & $\Delta$ SDR & $\Delta$ ISR & $\Delta$ SIR & $\Delta$ SAR & $\Delta$ OPS & $\Delta$ TPS & $\Delta$ IPS & $\Delta$ APS \\
\hline \multirow{2}{*}{ Vocal } & $\mathbf{1 . 2 6}$ & $\mathbf{1 . 3 0}$ & -6.90 & $\mathbf{1 2 . 7}$ & $\mathbf{0 . 0 8}$ & -0.04 & -0.09 & $\mathbf{0 . 5 6}$ \\
& $\mathbf{3 . 0 6}$ & $\mathbf{4 . 9 0}$ & -3.40 & $\mathbf{1 4 . 2}$ & $\mathbf{0 . 1 3}$ & $\mathbf{0 . 1 9}$ & -0.07 & $\mathbf{0 . 5 9}$ \\
Drums & -0.08 & -4.70 & -6.80 & $\mathbf{1 0 . 6}$ & $\mathbf{0 . 0 6}$ & $\mathbf{0 . 2 6}$ & -0.11 & -0.11 \\
& $\mathbf{7 . 7 2}$ & $\mathbf{1 1 . 0}$ & -4.10 & $\mathbf{1 6 . 7}$ & $\mathbf{0 . 0 9}$ & $\mathbf{0 . 5 9}$ & -0.05 & -0.05 \\
Guitar & $\mathbf{0 . 7 6}$ & -1.50 & -5.60 & $\mathbf{1 4 . 5}$ & $\mathbf{0 . 0 7}$ & $\mathbf{0 . 3 7}$ & -0.33 & $\mathbf{0 . 4 1}$ \\
& $\mathbf{4 . 7 6}$ & $\mathbf{9 . 3 0}$ & -1.30 & $\mathbf{1 8 . 1}$ & $\mathbf{0 . 1 1}$ & $\mathbf{0 . 4 7}$ & -0.38 & $\mathbf{0 . 5 6}$ \\
\hline \multirow{6}{*}{ Vocal } & $\mathbf{1 . 9 5}$ & -7.20 & -15.7 & $\mathbf{1 2 . 5}$ & $\mathbf{0 . 3 8}$ & -0.18 & $\mathbf{0 . 0 9}$ & $\mathbf{0 . 5 8}$ \\
& $\mathbf{3 . 5 5}$ & -0.60 & -11.8 & $\mathbf{1 4 . 3}$ & $\mathbf{0 . 5 3}$ & -0.14 & $\mathbf{0 . 1 1}$ & -0.16 \\
Drums & $\mathbf{0 . 6 5}$ & -2.64 & -6.33 & $\mathbf{1 1 . 0}$ & $\mathbf{0 . 0 4}$ & $\mathbf{0 . 0 4}$ & -0.17 & -0.40 \\
& $\mathbf{2 . 9 5}$ & $\mathbf{3 . 3 6}$ & -7.03 & $\mathbf{1 3 . 4}$ & $\mathbf{0 . 0 8}$ & $\mathbf{0 . 4 2}$ & -0.09 & $\mathbf{0 . 0 9}$ \\
Bass & -1.51 & -0.80 & -5.76 & $\mathbf{1 2 . 4}$ & -0.27 & $\mathbf{0 . 1 4}$ & -0.45 & $\mathbf{0 . 0 7}$ \\
& $\mathbf{5 . 1 9}$ & $\mathbf{1 8 . 0}$ & -4.66 & $\mathbf{2 2 . 4}$ & -0.15 & $\mathbf{0 . 7 4}$ & -0.35 & $\mathbf{0 . 0 7}$ \\
Claps & $\mathbf{1 . 3 2}$ & -5.80 & -17.5 & $\mathbf{1 3 . 1}$ & -0.69 & $\mathbf{0 . 1 8}$ & -0.22 & 0.00 \\
& $\mathbf{1 . 0 2}$ & -3.90 & -14.1 & $\mathbf{1 2 . 6}$ & -0.15 & $\mathbf{0 . 3 4}$ & -0.09 & -0.19 \\
\hline \multirow{2}{*}{ Vocal } & $\mathbf{2 . 5 7}$ & -7.60 & -14.7 & $\mathbf{1 6 . 2}$ & $\mathbf{0 . 3 1}$ & $\mathbf{0 . 1 3}$ & $\mathbf{0 . 0 5}$ & $\mathbf{0 . 2 5}$ \\
& $\mathbf{4 . 1 7}$ & -3.50 & -10.0 & $\mathbf{1 8 . 2}$ & $\mathbf{0 . 3 8}$ & $\mathbf{0 . 4 5}$ & $\mathbf{0 . 0 7}$ & $\mathbf{0 . 3 7}$ \\
Drums & $\mathbf{2 . 1 9}$ & -8.90 & -12.2 & $\mathbf{1 3 . 7}$ & $\mathbf{0 . 2 3}$ & -0.04 & -0.03 & $\mathbf{0 . 3 2}$ \\
& $\mathbf{6 . 0 9}$ & $\mathbf{9 . 6 0}$ & -8.40 & $\mathbf{1 7 . 7}$ & $\mathbf{0 . 2 5}$ & $\mathbf{0 . 5 8}$ & $\mathbf{0 . 0 1}$ & $\mathbf{0 . 3 0}$ \\
Bass & $\mathbf{2 . 5 2}$ & -4.60 & -14.8 & $\mathbf{1 4 . 3}$ & $\mathbf{0 . 0 3}$ & $\mathbf{0 . 1 7}$ & -0.30 & $\mathbf{0 . 3 0}$ \\
& $\mathbf{3 . 8 2}$ & $\mathbf{8 . 9 0}$ & -11.4 & $\mathbf{1 7 . 7}$ & -0.02 & $\mathbf{0 . 4 8}$ & -0.28 & $\mathbf{0 . 2 9}$ \\
\hline
\end{tabular}

Ultimate NZ Tour

Table 2. The differences between our scores and the scores reported in SiSEC 2011 for oracle systems. The upper row next to the track name represents the STFT-based system and the lower row represents the cochleagram-based system, respectively.

determined reverberant audio source separation using a full-rank spatial covariance model," IEEE Audio, Speech, Language Process., vol. 18, no. 7, pp. 18301840, Sep. 2010.

[3] S. Gorlow and S. Marchand, "Informed audio source separation using linearly constrained spatial filters," IEEE Audio, Speech, Language Process., vol. 21, no. 1, pp. 3-13, Jan. 2013.

[4] K. H. Knuth, "Informed source separation: A Bayesian tutorial," in Proc. EUSIPCO, Sep. 2005, pp. 1-8.

[5] A. Liutkus et al., "Informed source separation through spectrogram coding and data embedding," Signal Process., vol. 92, no. 8, pp. 1937-1949, Aug. 2012.

[6] ITU-T, Pulse code modulation (PCM) of voice frequencies, 1993, rec. ITU-T G.711.

[7] N. Sturmel et al., "Linear mixing models for active listening of music productions in realistic studio conditions," in AES Conv. 132, Apr. 2012, pp. 1-10.
[8] “SiSEC 2013,” http://sisec.wiki.irisa.fr.

[9] "The PEASS toolkit - Perceptual Evaluation methods for Audio Source Separation," http://bass-db.gforge. inria.fr/peass/, version 2.0.

[10] V. Emiya, E. Vincent, N. Harlander, and V. Hohmann, "Subjective and objective quality assessment of audio source separation," IEEE Audio, Speech, Language Process., vol. 19, no. 7, pp. 2046-2057, Sep. 2011.

[11] HörTech, "PEMO-Q," http://www.hoertech.de/web_en/ produkte/pemo-q.shtml, version 1.3.

[12] R. Huber and B. Kollmeier, "PEMO-Q - a new method for objective audio quality assessment using a model of auditory perception," IEEE Audio, Speech, Language Process., vol. 14, no. 6, pp. 1902-1911, Nov. 2006.

[13] “SiSEC 2011,” http://sisec2011.wiki.irisa.fr. 